\title{
Monte Carlo study of Dirac semimetals phase diagram
}

\author{
V. V. Braguta,,$^{1,2,3,4,{ }^{*}}$ M. I. Katsnelson, ${ }^{5,6, \dagger}$ A. Yu. Kotov, ${ }^{2,7, \ddagger}$ and A. A. Nikolaev ${ }^{2,3, \S}$ \\ ${ }^{1}$ Institute for High Energy Physics, NRC “Kurchatov Institute,” Protvino 142281, Russia \\ ${ }^{2}$ Institute of Theoretical and Experimental Physics, 117218 Moscow, Russia \\ ${ }^{3}$ School of Biomedicine, Far Eastern Federal University, 690950 Vladivostok, Russia \\ ${ }^{4}$ Moscow Institute of Physics and Technology, Institutskii pereulok 9, Dolgoprudny, Moscow Region 141700, Russia \\ ${ }^{5}$ Institute for Molecules and Materials, Radboud University, Heyendaalseweg 135, NL-6525 AJ Nijmegen, Netherlands \\ ${ }^{6}$ Theoretical Physics and Applied Mathematics Department, Ural Federal University, Mira Strasse 19, 620002 Ekaterinburg, Russia \\ ${ }^{7}$ Moscow Engineering Physics Institute, National Research Nuclear University, Kashirskoe Highway, 31, Moscow 115409, Russia
}

(Received 4 September 2016; revised manuscript received 4 November 2016; published 29 November 2016)

\begin{abstract}
In this paper the phase diagram of Dirac semimetals is studied within a lattice Monte Carlo simulation. In particular, we concentrate on the dynamical chiral symmetry breaking which results in a semimetal-insulator transition. Using numerical simulation, we determine the values of the critical coupling constant of the semimetalinsulator transition for different values of the anisotropy of the Fermi velocity. This measurement allows us to draw a tentative phase diagram for Dirac semimetals. It turns out that within the Dirac model with Coulomb interaction both $\mathrm{Na}_{3} \mathrm{Bi}$ and $\mathrm{Cd}_{3} \mathrm{As}_{2}$, known experimentally to be Dirac semimetals, would lie deep in the insulating region of the phase diagram. This result probably shows a decisive role of screening of the interelectron interaction in real materials, similar to the situation in graphene.
\end{abstract}

DOI: 10.1103/PhysRevB.94.205147

\section{INTRODUCTION}

Recent significant advances in condensed-matter physics are connected to the discovery of new materials with remarkable properties. Probably, the discovery of graphene $[1,2]$ is the most famous example. Graphene attracts considerable interest because of its unique electronic properties; most of them are related to the existence of two conical points in the electron energy spectrum (Fermi points) and massless fermion excitations, which are similar to two-dimensional (2D) Dirac fermions [3-7].

Recently, theory predicted [8,9] and, shortly afterwards, experiment confirmed the existence of so-called Dirac semimetals $\mathrm{Na}_{3} \mathrm{Bi}$ [10] and $\mathrm{Cd}_{3} \mathrm{As}_{2}$ [11,12], which manifest the properties of a three-dimensional (3D) analog of graphene. The low-energy spectrum of these materials is determined by two Fermi points. In the vicinity of each Fermi point the fermion excitations reveal the properties of massless 3D Dirac fermions with the dispersion relation

$$
E^{2}=v_{\|}^{2}\left(k_{x}^{2}+k_{y}^{2}\right)+v_{\perp}^{2} k_{z}^{2},
$$

where $v_{\|}, v_{\perp}$ are Fermi velocities in the $(x, y)$ plane and the $z$ direction, respectively. For $\mathrm{Na}_{3} \mathrm{Bi}, v_{\|} / c \simeq 0.001, v_{\perp} / v_{\|} \simeq$ 0.1 [10], and for $\mathrm{Cd}_{3} \mathrm{As}_{2}, v_{\|} / c \simeq 0.004, v_{\perp} / v_{\|} \simeq 0.25$ [13].

Due to the smallness of the Fermi velocities magnetic interactions and retardation effects can be safely disregarded. As a result the interaction in Dirac semimetals is reduced to the instantaneous Coulomb potential with the effective coupling constant $\alpha_{\text {eff }}=\alpha_{e l} c / v_{\|}>1$, where $\alpha_{e l}=1 / 137$. So one sees that the interaction is quite strong, which can dramatically modify the properties of these materials. In particular, it is

\footnotetext{
*braguta@itep.ru

†m.katsnelson@science.ru.nl

${ }^{\ddagger}$ kotov@itep.ru

§nikolaev.aa@dvfu.ru
}

known that strong interaction between quasiparticles can lead to dynamical chiral symmetry breaking, formation of the energy gap in the fermion spectrum, and transition from the semimetal to insulator phase.

This paper is devoted to the investigation of the phase diagram of Dirac semimetals. In particular, we are going to study the semimetal-insulator phase transition in the parameter plane $\left(\alpha_{\text {eff }}, v_{\perp} / v_{\|}\right)$, which results from dynamical chiral symmetry breaking at sufficiently strong interactions between quasiparticles. To carry out this study we are going to use lattice Monte Carlo simulation, which fully accounts for many-body effects in Dirac semimetals for an arbitrary coupling constant $\alpha_{\text {eff }}$. This approach proved to be very efficient in studying the properties of the strongly correlated systems, for instance, graphene [14-19]. We would like to stress that the advantage of our approach compared to the others is that one does not need to make any assumptions about the Dirac model. It should be noted that earlier, the phase diagram of Dirac semimetals was studied using the mean field [20,21], renormalization group [22,23], and Dyson-Schwinger equation [24].

Taking into account the spectrum of low-energy fermion excitations near the Fermi points and the properties of the interactions discussed above, the partition function of Dirac semimetals can be written in the following form:

$$
Z=\int D \psi D \bar{\psi} D A_{4} \exp \left(-S_{E}\right),
$$

where $\bar{\psi}, \psi$ are fermion fields and $A_{4}$ is the temporal component of the vector potential of the electromagnetic field. The Euclidean action $S_{E}$ can be written as

$$
\begin{aligned}
S_{E}= & \sum_{a=1}^{N_{f}=2} \int d^{3} x d t \bar{\psi}_{a}\left[\gamma_{4}\left(\partial_{4}+i A_{4}\right)+\xi_{i} \gamma_{i} \partial_{i}\right] \psi_{a} \\
& +\frac{1}{8 \pi \alpha_{\text {eff }}} \int d^{3} x d t\left(\partial_{i} A_{4}\right)^{2} .
\end{aligned}
$$


Here $\gamma_{1}, \ldots, \gamma_{4}$ are Euclidean gamma matrices, $\left\{\gamma_{\mu}, \gamma_{\nu}\right\}=$ $2 \delta_{\mu, \nu}$, and $\xi_{i}$ are factors which take into account the anisotropy of the Fermi velocity $\left(\xi_{1}=\xi_{2}=1, \xi_{3}=v_{\perp} / v_{\|}\right)$.

In Eq. (3) we rescaled $t$ and $A_{4}$, which allowed reabsorption of the Fermi velocity $v_{\|}$by $\alpha_{\text {eff }}$. As noted above, the smallness of the Fermi velocity $v_{\|} \ll c$ leads to the fact that the interaction between quasiparticles is described by the instantaneous Coulomb potential, which is transmitted by the field $A_{4}$. Partition function (2) does not depend on the vector part of the gauge potential $A_{i}$ since we are working at the leading approximation in $v_{\|}$.

\section{LATTICE FIELD THEORY FOR DIRAC SEMIMETALS}

In the lattice Monte Carlo approach one discretizes the continuum expression for the action (3). In our simulations we use staggered discretization for fermions [25] coupled to the Abelian lattice gauge field $\theta_{4}(x)$ :

$$
\begin{aligned}
S_{f}= & \bar{\Psi}_{x} D_{x, y} \Psi_{y}=\sum_{x}\left\{\operatorname{ma} \bar{\Psi}_{x} \Psi_{x}+\frac{1}{2}\left[\bar{\Psi}_{x} \eta_{4}(x) e^{i \theta_{4}(x)} \Psi_{x+\hat{4}}\right.\right. \\
& \left.-\bar{\Psi}_{x+4} \eta_{4}(x) e^{-i \theta_{4}(x)} \Psi_{x}\right] \\
& \left.+\frac{1}{2} \sum_{i=1}^{3} \xi_{i}\left[\bar{\Psi}_{x} \eta_{i}(x) \Psi_{x+\hat{\imath}}-\bar{\Psi}_{x+1} \eta_{i}(x) \Psi_{x}\right]\right\},
\end{aligned}
$$

where $\eta_{\mu}(x)=(-1)^{x_{0}+\cdots+x_{\mu-1}}, \mu=1, \ldots, 4$, are staggered factors corresponding to $\gamma$ matrices. The lattice field $\theta_{4}$ is related to the continuum Abelian field $A_{4}$ as $\theta_{4}=a A_{4}$, where $a$ is the lattice spacing. It should be noted that the nonzero mass term in (4) is necessary in order to ensure the invertibility of the staggered Dirac operator $D_{x, y}$. Physical results for zero mass are obtained by extrapolation of the expectation values of physical observables to the limit $m \rightarrow 0 .^{1}$

For discretization of the Abelian field the noncompact action was used:

$$
S_{g}=\frac{\beta}{2} \sum_{x, i}\left[\theta_{4}(x)-\theta_{4}(x+i)\right]^{2} .
$$

Here the constant $\beta$ is given by the formula $\beta=\frac{1}{4 \pi \alpha_{\mathrm{eff}}}$.

Integrating out fermion degrees of freedom, one gets the following expression for the partition function:

$$
\begin{aligned}
Z & =\int D \theta_{4}(x) \exp \left(-S_{\mathrm{eff}}\right), \\
S^{(\mathrm{eff})} & =-\ln \operatorname{det} D[\theta]+S_{g} .
\end{aligned}
$$

Notice, however, that effective action (6) in continuum corresponds to four degenerate fermion flavors [25] instead of the two observed in $\mathrm{Na}_{3} \mathrm{Bi}$ and $\mathrm{Cd}_{3} \mathrm{As}_{2}$. In order to get two fermion flavors we take the square root of the determinant of the Dirac operator that in the numerical simulation is realized through the rooting procedure. Thus the effective action used in the simulation is

$$
S^{(\mathrm{eff})}=-\frac{1}{2} \ln \operatorname{det} D[\theta]+S_{g} .
$$

\footnotetext{
${ }^{1}$ In this paper we express all dimensional observables in lattice units.
}

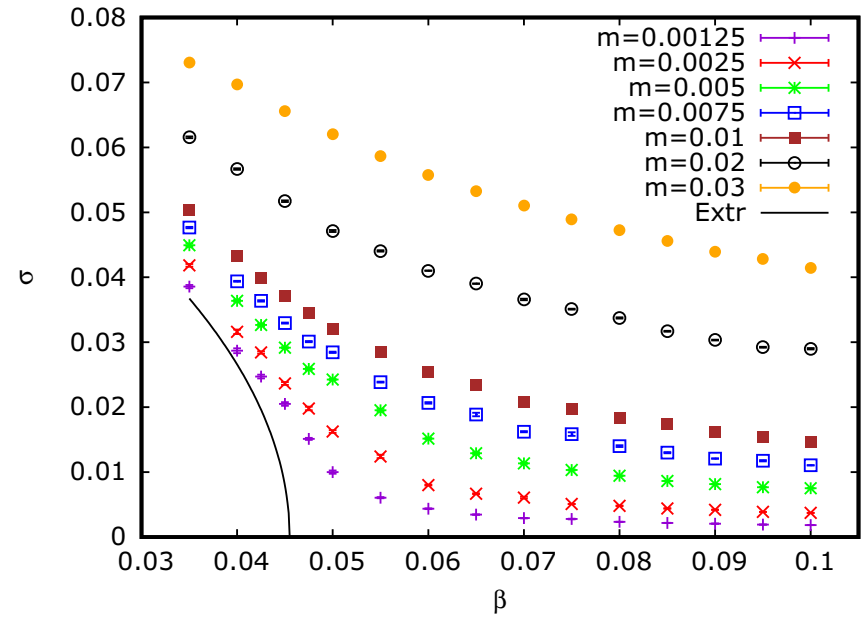

FIG. 1. The chiral condensate $\langle\bar{\Psi} \Psi\rangle$ as a function of $\beta$ for different values of mass $m$. Fermi velocity is isotropic $\xi_{1}=\xi_{2}=$ $\xi_{3}=1$. The black line corresponds to chiral limit $m \rightarrow 0$ taken with the help of EOS (8).

For generation of the field $\theta_{4}(x)$ with the statistical weight $\exp \left(-S^{(\mathrm{eff})}[\theta]\right)$ the standard hybrid Monte Carlo Method [25] was used.

As noted above, we are going to study the semimetalinsulator phase transition which is connected to dynamical chiral symmetry breaking. To determine the position of the phase transition we are going to measure the order parameter of chiral symmetry breaking, the chiral condensate $\sigma=\langle\bar{\Psi} \Psi\rangle$. In the chiral limit $m=0 ; \sigma=0$ in the chiral symmetric phase, and $\sigma \neq 0$ in the phase where chiral symmetry is broken.

In addition to the chiral condensate we will calculate the susceptibility of the chiral condensate $\chi_{L}=\frac{\partial \sigma}{\partial m}$. The observable related to the susceptibility and sensitive to the semimetal-insulator phase transition is the logarithmic derivative of the chiral condensate $R=\frac{\partial \ln \sigma}{\partial \ln m}$. In the chiral limit $R$ reveals the following properties: in the chirally symmetric phase $\sigma \sim m$ and $R \rightarrow 1$. At the critical point $R \rightarrow 1 / \delta$, where $\delta$ is a universal critical exponent, and $R \rightarrow 0$ in the phase with broken chiral symmetry.

\section{NUMERICAL RESULTS}

First, let us study the case without anisotropy of the Fermi velocity in different directions $\left(\xi_{1}=\xi_{2}=\xi_{3}=1\right)$. In numerical simulation of the Dirac semimetals we used a $20^{4}$ lattice. In Fig. 1 the dependence of $\sigma$ on $\beta$ for different fermion masses is presented. It is seen from this plot that the formation of the chiral condensate takes place at values of $\beta<\beta_{c}$, with the critical value $\beta_{c} \sim 0.04-0.06$.

In order to confirm this result we also studied the susceptibility of the chiral condensate $\chi_{L}=\frac{\partial \sigma}{\partial m}$ (Fig. 2) as a function of $\beta$ for different values of mass. The plot shows a clear peak at small values of mass $m \leqslant 0.005$, which is also an indication of the phase transition. The critical value of $\beta_{c}$ determined from the position of the peak is slightly larger and decreases when the mass decreases. This behavior is expected because it is well known that nonzero mass shifts the position of the transition to larger values of $\beta$. 


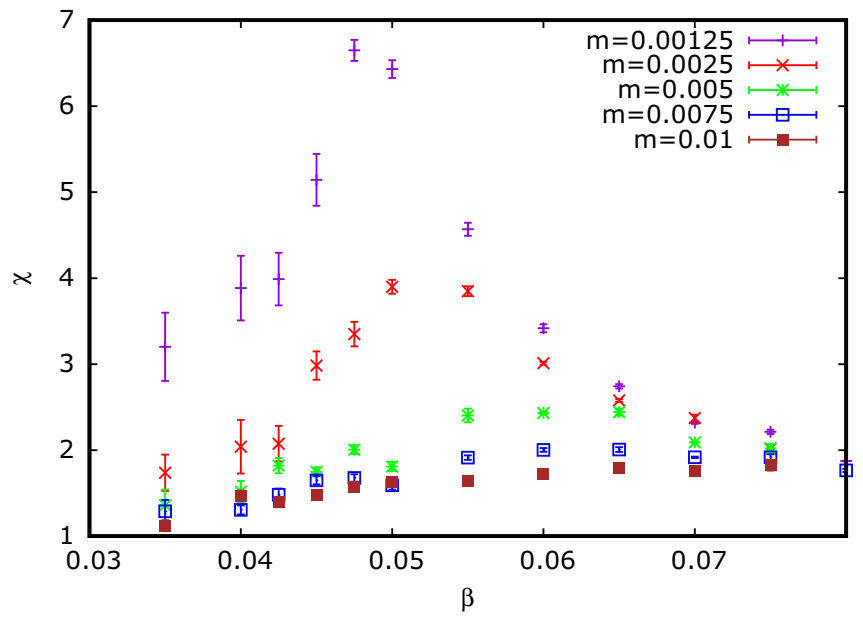

FIG. 2. Susceptibility $\chi$ of the chiral condensate as a function of $\beta$ for different values of mass $m$. Fermi velocity is isotropic, $\xi_{1}=\xi_{2}=\xi_{3}=1$.

In Fig. 3 we plot $R$ as a function of $m$ for different values of $\beta$. Taking into account the properties of $R$ discussed above, one can conclude that for $\beta \geqslant 0.0475$ the system has no gap, while for $\beta \leqslant 0.0425$ the results indicate the formation of the gap in the chiral limit. It allows us to estimate the critical value of the coupling $\beta=0.0450 \pm 0.0025$, which is in agreement with the estimation of $\beta_{c}$ from the data for the condensate.

To estimate the values of $\beta_{c}$ more precisely we fit the data with an equation of state (EOS) $m=f(\sigma, \beta)$. Motivated by studies of QED [26] and graphene [14], we apply the following equation of state:

$$
m X(\beta)=Y(\beta) f_{1}(\sigma)+f_{3}(\sigma),
$$

where one expands $X(\beta)=X_{0}+X_{1}\left(1-\beta / \beta_{c}\right)$ and $Y(\beta)=$ $Y_{1}\left(1-\beta / \beta_{c}\right)$ in the vicinity of critical $\beta_{c}$. For the left-hand side we used classical critical exponents: $f_{1}(\sigma)=\sigma, f_{3}(\sigma)=\sigma^{3}$. Such an EOS can be easily visualized if one plots $\sigma^{2}$ as a function of $m_{0} / \sigma$ for various values of $\beta$ (Fisher plot). The

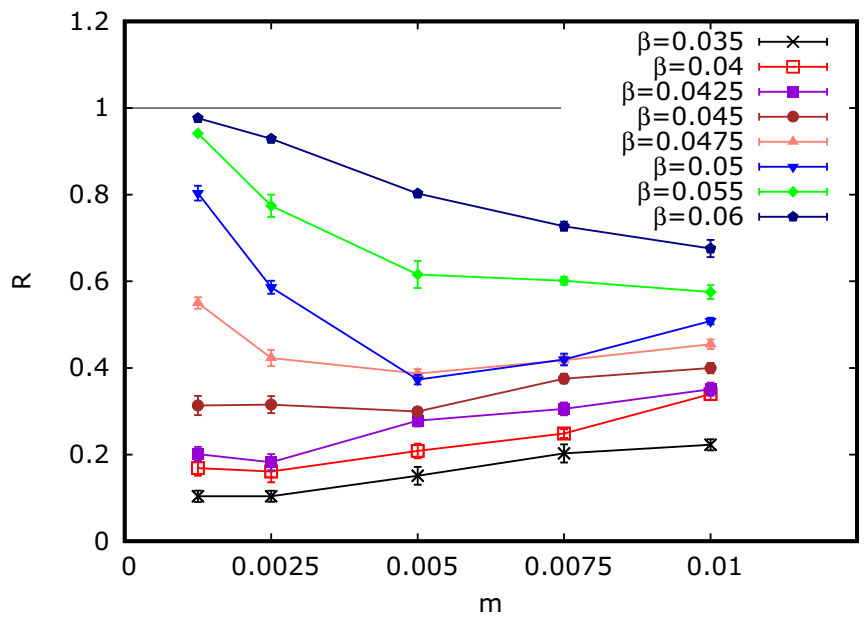

FIG. 3. Logarithmic derivative of $R$ of the chiral condensate as a function of mass $m$ for different values of the coupling constant $\beta$. Fermi velocity is isotropic, $\xi_{1}=\xi_{2}=\xi_{3}=1$.

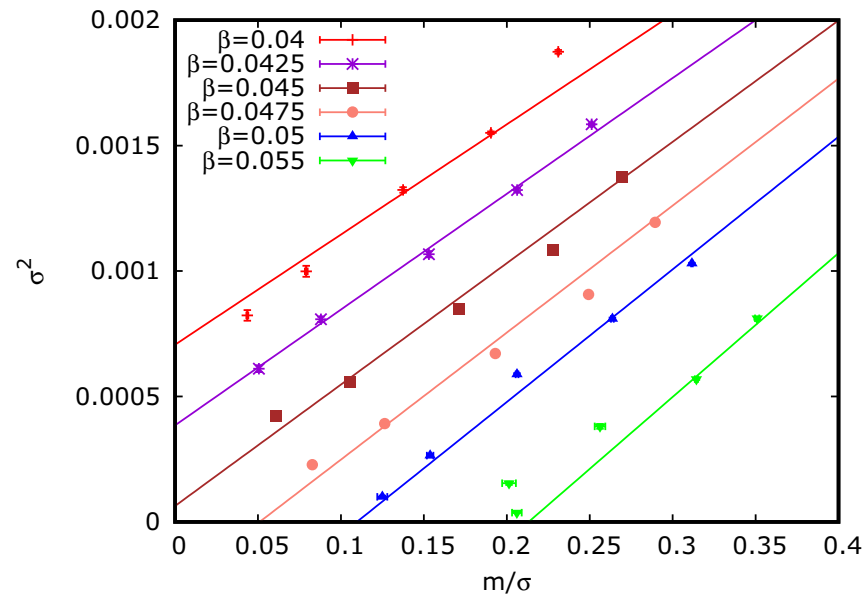

FIG. 4. The square chiral condensate $\sigma$ as a function of $\frac{m}{\sigma}$. Fermi velocity is isotropic, $\xi_{1}=\xi_{2}=\xi_{3}=1$. Straight lines correspond to the fit of all points with EOS (8).

resulting dependence $\sigma^{2}\left(m_{0} / \sigma\right)$ forms straight lines, crossing the origin at $\beta_{c}$. This Fisher plot is presented in Fig. 4. The deviations from straight lines might be attributed to finitevolume effects or to nonclassical critical exponents. The fit of the data in the vicinity of transition using Eq. (8) is given by the straight lines in Fig. 4. Using this fit, we obtained $\beta_{c}=$ $0.04549(6)$. The presented error is only statistical. This value of $\beta_{c}$ corresponds to the critical coupling $\alpha_{\mathrm{eff}}^{c}=1.749(2)$.

It would be interesting to discuss other approaches which were applied to study the phase diagram of Dirac semimetals and to compare our results with them. In [20] the properties of Dirac semimetals were studied at infinitely large coupling $(\beta=$ 0 ) and within the mean field. The authors drew the tentative phase diagram of the system and found that in the isotropic case the system is gapless, while it appears to have a gap if the anisotropy of the Fermi velocity $v_{\perp} / v_{\|}$is smaller than some critical value $\xi_{3} \sim 0.25$. The authors of [21] showed that some important points were missed in [20], and they improved this method. It was shown that in the limit of infinitely strong coupling $\beta=0$ for all values of Fermi velocity anisotropy there is a gap. In [24] this problem was studied by means of Dyson-Schwinger equations. The critical value of the coupling constant in the isotropic case was found to be much larger $\alpha \approx 14.7$. Later, the same authors revised this result within the ladder approximation [22] and found the critical coupling to be $\alpha_{\text {eff }}^{c}=1.8660$, which is close to the critical coupling $\alpha_{\text {eff }}^{c}=1.749(2)$ obtained in this paper.

Having accomplished the study of isotropic Dirac semimetals, we proceed to the anisotropic case, which is parametrized by the value $\xi_{3}=\xi<1\left(\xi_{1}=\xi_{2}=1\right)$. The study was conducted on a $20^{4}$ lattice using the procedure described above for the values of $\xi=0.1,0.2,0.5$. For these values of $\xi$ the results are similar to those in Figs. 1-4 for the isotropic case. For that reason we do not show them here. We have found the following values of critical $\beta_{c}: \alpha_{\text {eff }}^{c}=1.762(3)$ for $\xi=0.5$, $\alpha_{\text {eff }}^{c}=1.467(10)$ for $\xi=0.2$, and $\alpha_{\text {eff }}^{c}=1.150(8)$ for $\xi=0.1$. So we see that at $\xi=0.5, \beta_{c}$ is practically the same as that at $\xi=1$. For $\xi \leqslant 0.2$ the value of $\beta_{c}$ quickly increases with the decrease in $\xi$. A tentative phase diagram is shown in Fig. 5. 


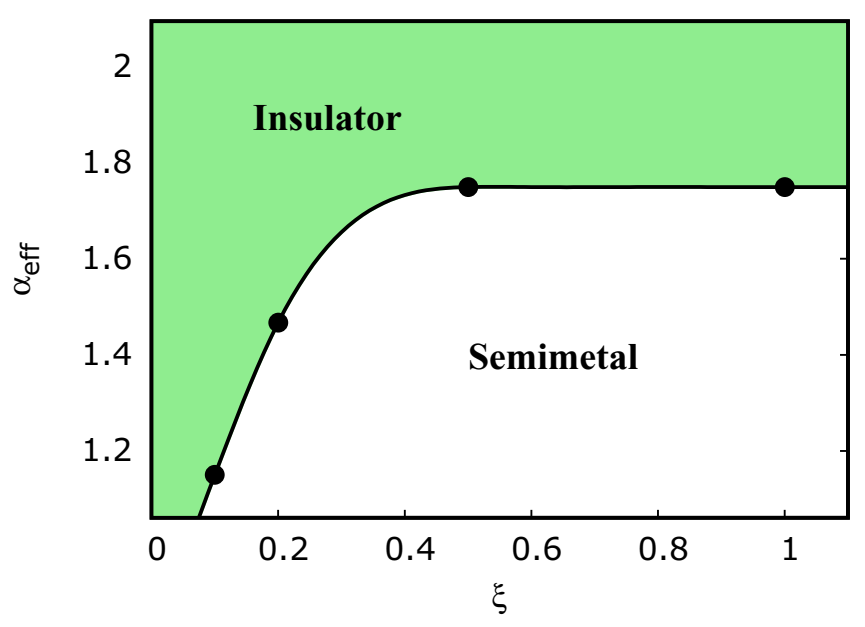

FIG. 5. The dependence of the critical coupling constant $\alpha_{\mathrm{eff}}^{c}$ on the Fermi velocity anisotropy $\xi$. For $\alpha_{\text {eff }}>\alpha_{\text {eff }}^{c}(\xi)$ the system is in the insulator phase. Smaller values of $\alpha_{\text {eff }}<\alpha_{\text {eff }}^{c}(\xi)$ correspond to the semimetal phase. Statistical errors are smaller than data points. Lines are to guide the eyes.

Note that the parameter $\xi$ effectively controls the dimension of the system under study. In the isotropic case $\xi=1$ the system is three-dimensional. At $\xi=0$ the system is similar to a stack of two-dimensional sheets with Fermi velocity $v_{\|}$. From quantum mechanics one may expect the critical coupling for the 2D system to be smaller than that for the 3D system, which is in agreement with the phase diagram in Fig. 5.

A detailed analysis of finite-volume effects requires considerable computational resources, and it will be done in a separate study. However, in order to estimate the volume dependence of our results we carried out lattice simulation of Dirac semimetals on a $24^{4}$ lattice for the asymmetries $\xi=0.1$ and $\xi=1$. For $\xi=0.1$ the critical coupling increases by $5 \%$, and for $\xi=1$ the critical coupling increases by $10 \%$. So this shows that the volume dependence will not change our results dramatically.

According to the experimental results, the effective coupling constants for the Dirac semimetals $\mathrm{Na}_{3} \mathrm{Bi}$ and $\mathrm{Cd}_{3} \mathrm{As}_{2}$ are $\alpha_{\text {eff }} \simeq 7$ and $\alpha_{\text {eff }} \simeq 2$, respectively. The analysis carried out in this paper implies that $\mathrm{Na}_{3} \mathrm{Bi}$ and $\mathrm{Cd}_{3} \mathrm{As}_{2}$ are deep in the insulator phase, which contradicts the experiments. So our paper raises a very important question about the theory of Dirac semimetals: Why does such strong interaction in Dirac semimetals not lead to dynamical generation of the energy gap in the fermion spectrum? A possible resolution of this puzzle is that in the real world the interaction potential is screened by bound electrons, which was not accounted for in our study. We believe that the mechanism which is possibly behind this phenomenon is similar to what was observed in graphene [18]. In graphene bound electrons considerably diminish the interaction potential at small distances. Thus the critical coupling of the phase transition shifts to larger values. Another possible explanation is that due to the renormalization effects strong interaction can considerably modify the basic parameters of the theory. Although the study of the different answers to this question is very important, it is beyond the scope of this paper.

\section{CONCLUSIONS}

In this paper the phase diagram of Dirac semimetals was studied within the lattice Monte Carlo simulation. In particular, we concentrated on the dynamical chiral symmetry breaking which results in a semimetal-insulator transition. We measured the chiral condensate and the susceptibility of the chiral condensate for different values of the fermions mass, the effective coupling constant, and the anisotropy of the Fermi velocity. Using these measurements, we determined the values of the critical coupling constant of the semimetal-insulator transition for different values of the anisotropy of the Fermi velocity. This measurement allowed us to draw a tentative phase diagram of Dirac semimetals.

It turns out that within the Dirac model with Coulomb interaction both $\mathrm{Na}_{3} \mathrm{Bi}$ and $\mathrm{Cd}_{3} \mathrm{As}_{2}$, known experimentally to be Dirac semimetals, would lie deep in the insulating region of the phase diagram. This result probably shows a decisive role of screening of the interelectron interaction in real materials, similar to the situation in graphene.

\section{ACKNOWLEDGMENTS}

The authors would like to express their gratitude to M. A. Zubkov, who drew their attention to the problem considered in this paper. The authors thank I. A. Shovkovy and Z. V. Khaidukov for useful discussions and comments. The work of M.I.K. was supported by Act 211 of the government of the Russian Federation, Contract No. 02.A03.21.0006. The work of V.V.B. and A.Y.K., which consisted of numerical simulation and the determination of the critical coupling constants at different Fermi velocity asymmetries, was supported by a grant from the Russian Science Foundation (Project No. 16-12-10059). Numerical simulations were carried out on the GPU cluster of the National Research Center "Kurchatov Institute".
[1] K. S. Novoselov, A. K. Geim, S. V. Morozov, D. Jiang, Y. Zhang, S. V. Dubonos, I. V. Grigorieva, and A. A. Firsov, Science 306, 666 (2004).

[2] A. K. Geim and K. S. Novoselov, Nat. Mater. 6, 183 (2007).

[3] P. R. Wallace, Phys. Rev. 71, 622 (1947).

[4] J. W. McClure, Phys. Rev. 104, 666 (1956).

[5] G. W. Semenoff, Phys. Rev. Lett. 53, 2449 (1984).
[6] K. S. Novoselov, A. K. Geim, S. V. Morozov, D. Jiang, M. I. Katsnelson, I. V. Grigorieva, S. V. Dubonos, and A. A. Firsov, Nature (London) 438, 197 (2005).

[7] Y.Zhang, Y.-W. Tan, H. L. Stormer, and P. Kim, Nature (London) 438, 201 (2005).

[8] Z. Wang, Y. Sun, X.-Q. Chen, C. Franchini, G. Xu, H. Weng, X. Dai, and Z. Fang, Phys. Rev. B 85, 195320 (2012). 
[9] Z. Wang, H. Weng, Q. Wu, X. Dai, and Z. Fang, Phys. Rev. B 88, 125427 (2013).

[10] Z. K. Liu, B. Zhou, Y. Zhang, Z. J. Wang, H. M. Weng, D. Prabhakaran, S.-K. Mo, Z. X. Shen, Z. Fang, X. Dai, Z. Hussain, and Y. L. Chen, Science 343, 864 (2014).

[11] M. Neupane, S.-Y. Xu, R. Sankar, N. Alidoust, G. Bian, C. Liu, I. Belopolski, T.-R. Chang, H.-T. Jeng, H. Lin, A. Bansil, F. Chou, and M. Z. Hasan, Nat. Commun. 5, 3786 (2014).

[12] S. Borisenko, Q. Gibson, D. Evtushinsky, V. Zabolotnyy, B. Büchner, and R. J. Cava, Phys. Rev. Lett. 113, 027603 (2014).

[13] Z. K. Liu, J. Jiang, B. Zhou, Z. J. Wang, Y. Zhang, H. M. Weng, D. Prabhakaran, S.-K. Mo, H. Peng, P. Dudin, T. Kim, M. Hoesch, Z. Fang, X. Dai, Z. X. Shen, D. L. Feng, Z. Hussain, and Y. L. Chen, Nat. Mater. 13, 677 (2014).

[14] J. E. Drut and T. A. Lahde, Phys. Rev. Lett. 102, 026802 (2009).

[15] S. Hands and C. Strouthos, Phys. Rev. B 78, 165423 (2008).
[16] W. Armour, S. Hands, and C. Strouthos, Phys. Rev. B 81, 125105 (2010).

[17] J. E. Drut and T. A. Lahde, Phys. Rev. B 79, 165425 (2009).

[18] M. V. Ulybyshev, P. V. Buividovich, M. I. Katsnelson, and M. I. Polikarpov, Phys. Rev. Lett. 111, 056801 (2013).

[19] D. L. Boyda, V. V. Braguta, M. I. Katsnelson, and M. V. Ulybyshev, Phys. Rev. B 94, 085421 (2016).

[20] A. Sekine and K. Nomura, Phys. Rev. B 90, 075137 (2014).

[21] Y. Araki, http://pos.sissa.it/cgi-bin/reader/contribution. cgi?id=251/046.

[22] J. Gonzalez, J. High Energy Phys. 10 (2015) 190.

[23] B. Roy and S. Das Sarma, Phys. Rev. B 94, 115137 (2016).

[24] J. Gonzalez, Phys. Rev. B 92, 125115 (2015).

[25] I. Montvay and G. Munster, Quantum Fields on a Lattice (Cambridge University Press, Cambridge, 1997).

[26] M. Gockeler, R. Horsley, V. Linke, P. E. L. Rakow, G. Schierholz, and H. Stuben, Nucl. Phys. B 487, 313 (1997). 